\title{
Mejora de las propiedades del lodo de EDAR mediante torrefacción en un reactor de tornillo sinfín
}

\author{
M. Atienza-Martínez, J.F. Mastral, J. Ábrego, J. Ceamanos, G. Gea \\ Grupo de Procesos Termoquímicos (GPT) \\ Instituto de Investigación en Ingeniería de Aragón (I3A). \\ Universidad de Zaragoza, Mariano Esquillor s/n, 50018, Zaragoza, Spain. \\ Tel.+34-976762707, Fax +34-976762043, e-mail: atienza@unizar.es
}

\section{Resumen}

En el presente trabajo se estudia la torrefacción de lodo de EDAR como pretratamiento para su posterior uso como materia prima en otros procesos termoquímicos. Bajo determinadas condiciones, se ha conseguido disminuir el contenido en oxígeno y aumentar la densidad energética del lodo.

\section{Introducción}

En los últimos años ha aumentado la cantidad de lodo de estaciones depuradoras de aguas residuales (EDAR) generada debido a una legislación sobre tratamiento de aguas cada vez más restrictiva. En España se planteó que en el año 2015 el 18 \% del lodo generado fuera valorizado de forma alternativa a su aplicación en suelos [1]. Procesos termoquímicos como la pirólisis y la gasificación supondrían la valorización energética de este residuo [2]. En general, la biomasa tiene una serie de propiedades, como por ejemplo su elevada humedad, su alto contenido en oxígeno y su baja densidad energética, que hacen necesario pretratarla antes de someterla a otros procesos de obtención de energía. Entre los posibles pretratamientos destaca la torrefacción, un proceso termoquímico llevado a cabo a temperaturas entre $200{ }^{\circ} \mathrm{C}$ y $300{ }^{\circ} \mathrm{C}$ en atmósfera inerte [3]. En la torrefacción se obtienen tres productos: sólido torrefactado, vapores condensables y gases no condensables. El objetivo de este pretratamiento es obtener un sólido con menor contenido en humedad, menores ratios molares $\mathrm{O} / \mathrm{C}$ y H/C, y mayor densidad energética. Los estudios sobre torrefacción de lodo de EDAR son todavía escasos. Se ha llevado a cabo la torrefacción de este material en mufla [4], en reactor de lecho fijo [5] y de lecho fluidizado [5, 6], pero no en reactores de tornillo sinfín, como se plantea en el presente trabajo. El objetivo es estudiar el efecto de las condiciones de torrefacción de lodo de EDAR en un reactor de tonillo sinfín sobre el rendimiento a producto sólido y sobre sus propiedades.
En la Tabla 1 se muestran las propiedades del lodo de EDAR digerido anaeróbicamente y secado térmicamente empleado en este estudio.

Tabla 1. Propiedades del lodo de partida.

\begin{tabular}{|l|l|l|l|}
\hline Materia seca (\%) & 93.5 & Carbono (\%) & 29.5 \\
\hline Cenizas (\%) & 39.0 & Hidrógeno (\%) & 4.7 \\
\hline Volátiles (\%) & 50.1 & Nitrógeno (\%) & 5.3 \\
\hline Carbono fijo (\%) & 4.4 & Azufre (\%) & 1.3 \\
\hline PCS (MJ·kg ${ }^{-1}$ ) & 12.8 & Oxígeno (\%) & 20.20 \\
\hline
\end{tabular}

Los experimentos de torrefacción se han llevado a cabo a diferentes temperaturas $\left(250{ }^{\circ} \mathrm{C}, 275{ }^{\circ} \mathrm{C}\right.$ y $300{ }^{\circ} \mathrm{C}$ ) y diferentes tiempos de residencia (13.3 $\mathrm{min}, 24.0 \mathrm{~min}$ y $34.7 \mathrm{~min}$ ) en un reactor de tornillo sinfín a escala de laboratorio. Se han recogido, cuantificado y caracterizado los tres productos obtenidos en los diferentes experimentos, siendo el sólido el producto de interés. El rendimiento a lodo torrefactado $\left(\eta_{\mathrm{LT}}\right)$ se ha calculado según la Ecuación 1:

$\eta_{\mathrm{LT}}(\%$ masa $)=100 \cdot \frac{\operatorname{masa}_{\mathrm{LT}}(\mathrm{g})}{\text { masa }_{\text {lodo alimentado }}(\mathrm{g})}$

Se han determinado, entre otros, el poder calorífico superior $\left(\mathrm{PCS}_{\mathrm{LT}}\right)$ y la densidad $\left(\rho_{\mathrm{LT}}\right)$ de los sólidos torrefactados para poder calcular el rendimiento energético ( $\eta_{\text {energético }}$ ) según la Ecuación 2 y la densidad energética $\left(\rho_{\text {energética }}\right)$ según la Ecuación 3 :

$$
\begin{aligned}
& \eta_{\text {energético }}(\%)=\eta_{\mathrm{LT}} \cdot \frac{\mathrm{PCS}_{\mathrm{LT}}\left(\mathrm{MJ} \cdot \mathrm{kg}^{-1}\right)}{\mathrm{PCS}_{\text {lodo seco }}\left(\mathrm{MJ} \cdot \mathrm{kg}^{-1}\right)} \quad \text { (Ec. 2) } \\
& \rho_{\text {energética }}\left(\mathrm{MJ} \cdot \mathrm{dm}^{-3}\right)=\rho_{\mathrm{LT}}\left(\mathrm{kg} \cdot \mathrm{dm}^{-3}\right) \cdot \mathrm{PCS}_{\mathrm{LT}}\left(\mathrm{MJ} \cdot \mathrm{kg}^{-1}\right)
\end{aligned}
$$

\section{Materiales y métodos}




\section{Resultados}

El rendimiento a lodo torrefactado disminuye al aumentar tanto la temperatura de torrefacción como el tiempo de residencia y esto provoca un fuerte descenso del rendimiento energético con la severidad de la torrefacción (Figura 1). En la Figura 2 se observa que cuando la torrefacción se lleva a cabo a temperaturas altas $\left(275{ }^{\circ} \mathrm{C}\right.$ o mayor) y tiempos de residencia largos (24.0 min o mayor) la densidad energética es menor que la del lodo de partida. Únicamente en las condiciones de torrefacción más suaves del presente trabajo $\left(250^{\circ} \mathrm{C}\right.$ y $13.3 \mathrm{~min}$ ) se consigue mejorar la densidad energética del lodo. Estos resultados difieren de los obtenidos por otros autores en el caso de la biomasa lignocelulósica, ya que este lodo de EDAR tiene un alto contenido en cenizas que aumenta con la severidad de la torrefacción y hace que el $\mathrm{PCS}_{\mathrm{LT}}$ disminuya. Tanto el ratio molar $\mathrm{O} / \mathrm{C}$ como el $\mathrm{H} / \mathrm{C}$ disminuyen con la severidad de la torrefacción (Figura 3) ya que se pierde dióxido de carbono y agua (tanto la contenida inicialmente en el lodo como la generada mediante reacciones de deshidratación) durante este proceso.

\section{Conclusiones}

De los resultados del presente trabajo se desprende que bajo las condiciones de torrefacción más suaves es posible disminuir el contenido en oxígeno del lodo de EDAR mejorando a su vez su densidad energética $\mathrm{y}$ consiguiendo un rendimietno energético por encima del $90 \%$. La torrefacción muy severa provoca una notable reducción del contenido en oxígeno, pero a costa de una reducción en la densidad energética y un bajo rendimiento másico y energético.

\section{REFERENCIAS}

[1]. ESPAÑA, MINISTERIO DE MEDIO AMBIENTE Y MEDIO RURAL Y MARINO. Resolución de 20 de enero de 2009, de la Secretaría de Estado de Cambio Climático, por la que se [...] aprueba el Plan Nacional Integrado de Residuos para el período 2008-2015. Boletín Oficial del Estado, 26 de febrero de 2009, núm. 49, pp. 19893-20016.

[2]. WERTHER, J. and OGADA, T. Sewage sludge combustion. Progress in Energy and Combustion Science. 1999, 25(1), 55-116. Available from: doi: 10.1016/S03601285(98)00020-3.

[3]. BASU, P. Biomass Gasification, Pyrolysis and Torrefaction. Practical Design and Theory. 2nd Edition. Amsterdam: Elsevier, 2013.
[4]. DHUNGANA, A., DUTTA, A. and BASU, P. Torrefaction of non -lignocellulose biomass waste. Canadian Journal of Chemical Engineering, 2012, 90 (1), 186-195. Available from: doi:10.1001/cjce.20257.

[5]. ÁBREGO, J., SÁNCHEZ, J.L., ARAUZO, J., FONTS, I., GIL-LALAGUNA, N. and ATIENZA-MARTÍNEZ, M. Technical and energetic assessment of a three-stage thermochemical treatment for sewage sludge. Energy \& Fuels, 2013, 27(2), 1026-1034. Available from: doi:10.1021/ef3018095

[6]. ATIENZA-MARTÍNEZ, M., FONTS, I., ÁBREGO, J., CEAMANOS, J. and GEA, G. Sewage sludge torrefaction in a fluidized bed reactor. Chemical Engineering Journal, 2013, 222, 534-545. Available from: doi:10.1016/j.cej.2013.02.075.

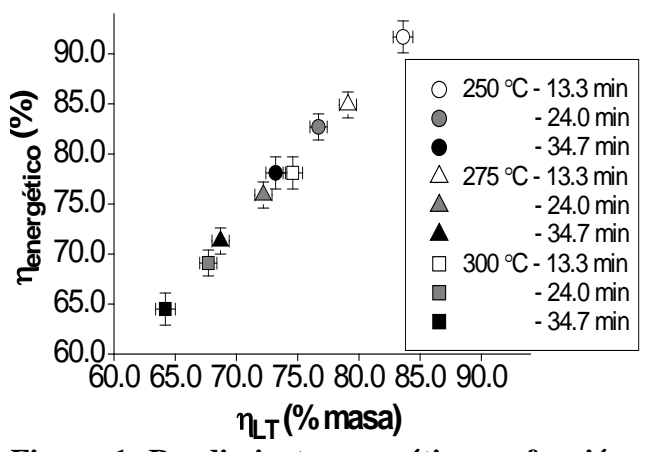

Figura 1. Rendimiento energético en función del rendimiento másico a lodo torrefactado.

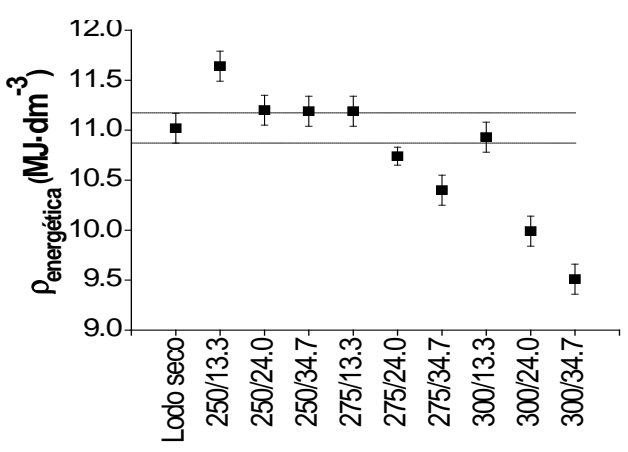

Figura 2. Comparación de la densidad energética del lodo seco y el LT en distintas condiciones.

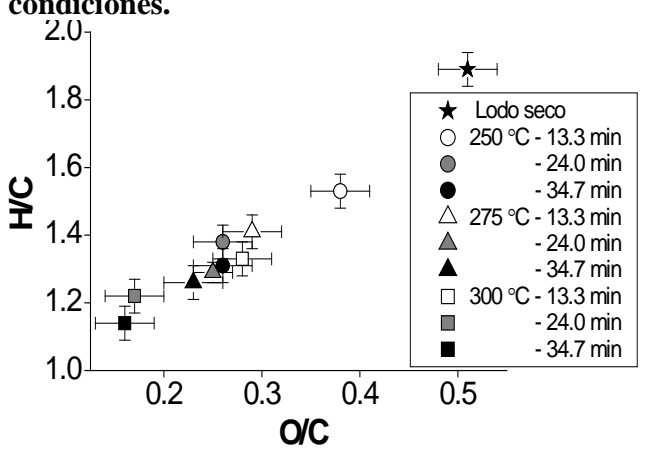

Figura 3. Diagrama de Van Krevelen. 